\title{
Effect of drying techniques on the retention of antioxidant activities of Saskatoon berries
}

\author{
Pranabendu Mitra $^{\mathrm{a}^{*}}$, Venkatesh Meda $^{\mathrm{a}}$, And Rick Green ${ }^{\mathrm{b}}$ \\ ${ }^{\text {a }}$ Department of Chemical and Biological Engineering, University of Saskatchewan, Saskatoon, SK, S7N 5A9, \\ Canada \\ b POS Bio-Sciences, 118 Veterinary Road, Saskatoon, SK, S7N 2R4, Canada \\ ${ }^{*}$ Corresponding author \\ pranabendumitra@yahoo.com \\ TEL: (+1-306) 966-5309 FAX: (+1-306) 966-5334
}

Received: 27 January 2013; Published online: 18 October 2013

\begin{abstract}
The main objective of this research was to compare the retention of antioxidant activity and total anthocyanin content of Saskatoon berries dried by freeze drying, microwave-vacuum drying, thin layer hot air drying and vacuum drying. Antioxidant activity of berry samples was determined by DPPH radical scavenging and ABTS radical scavenging, and the $\mathrm{pH}$ differential method was used to determine total anthocyanin content of the berry samples. The results showed that the freeze dried Saskatoon berries exhibited the highest retention of anthocyanin and antioxidant activity among the dried samples, followed by microwave-vacuum dried berries, thin layer hot air dried berries and vacuum dried berries. There were significant differences between the berry samples at $\mathrm{P}<0.05$. DPPH radical scavenging and ABTS radical scavenging were correlated linearly with an $\mathrm{R}^{2}$ value of 0.99 at $\mathrm{P}<0.05$ showing their effectiveness for the determination of the antioxidant activity of the Saskatoon berries. However, the DPPH radical scavenging assay was more effective than the ABTS radical scavenging assay. The results also showed that antioxidant activity of the berries was highly correlated with the total anthocyanin content of the fruit. The reduction of anthocyanin in dried berry samples was linearly correlated with the reduction of DPPH radical scavenging with an $\mathrm{R}^{2}$ value of 0.97 at $\mathrm{P}<0.05$ and, also, linearly correlated with the reduction of ABTS radical scavenging with an $\mathrm{R}^{2}$ value of 0.88 at $\mathrm{P}<0.05$.
\end{abstract}

Keywords: Saskatoon berries drying; antioxidant activity retention; anthocyanin; DPPH; ABTS; radical scavenging

\section{Introduction}

The consumption potentiality of Saskatoon berries (Amelanchier alnifolia) was started by the native Canadian and Northern people and the early settlers in these areas. These people consumed these berries as one of their key food sources and they also used Saskatoon berries to treat different ailments, for example, stomach, eye and ear ailments (Hu, Kwok, \& Kitts, 2005;
Mazza \& Cottrell, 2008). Some studies showed that these berries contained significant amounts of minerals (potassium, iron, magnesium and phosphorous) and vitamins (vitamin $\mathrm{C}$, thiamin, riboflavin, pantothenic acid, vitamin B6, vitamin A and vitamin E) (Mazza, 1982; Mazza \& Cottrell, 2008; Ozga, Saeed, \& Reinecke, 2006). The reported high levels of anthocyanins and phenolic compounds in Saskatoon berries impart free radical scavenging properties and thus, these berries 
are a potentially good source of antioxidants $(\mathrm{Hu}$ et al., 2005; Lavola, Karjalainen, \& JulkunenTiitto, 2012; Mazza, 1986). There is increasing interest in Saskatoon berries as fresh whole fruit and because jam, jelly, chocolates and juice are being produced from this fruit on a commercial scale (Lavola et al., 2012; Mitra \& Meda, 2009). However, the fresh berries possess a very short shelf life due to their high moisture content (80-84\%). Drying the berries is needed in order to increase their shelf life and to preserve them for transportation and utilization throughout the year.

Drying can be defined simply as a method of safe level moisture content reduction of products to minimize the deterioration of the quality of products to enable longer term storage and to increase the economic return. In practice, there are many methods of drying such as freeze drying, microwave-vacuum drying, vacuum drying and hot air drying, etc. Although the purpose of all drying methods is the same, the heat generation and heat transfer phenomena to the materials are quite different from each other (Dev \& Raghavan, 2012; Ratti, 2001). Freeze drying is one of the best drying methods to retain the quality of dried products; however, this method is relatively expensive for commercial drying of fruits. Freeze drying occurs under a very low temperature whereby the solid (i.e. frozen) state of water in the fruit changes directly to the gaseous state by sublimation. The low drying temperature and absence of liquid water during drying retard most biochemical and microbiological deteriorations, and the solid state of water during freeze drying retains the primary structure and shape of the products with minimal reduction of volume. Microwave drying is a technique whereby electromagnetic waves (i.e. microwaves) transfer through materials and oscillate molecules to generate heat. The microwaves generate heat within the entire volume of the materials at about the same rate. Microwave drying combined with vacuum improves color, texture, flavor and nutrient retention of dried products because of volumetric heating and shorter drying times. In addition, the vacuum helps to reduce drying temperature by reducing the boiling point of water during microwave-vacuum drying. The most common frequently used conventional drying method is hot air drying. In this process, the surface of the ma- terials is exposed to hot air and the heat transfers from the surface to the inside of the materials. The rate of water evaporation is faster than the mass transfer of water to the product surface due to rapid drying of surface of the products and uneven heat transfer into the products. Materials are exposed to a relatively high drying temperature for a relatively long residence time in the hot air drier. Also, since materials are exposed to air in the drier enzymatic and non-enzymatic browning reactions may occur. Vacuum drying can be performed at a low temperature and this drying system is suitable for heat sensitive materials. The vacuum drying can result in greater retention of nutrients as well as volatile compounds in the dried products, but this drying method is limited in use because of higher costs and prolonged drying time (Dev \& Raghavan, 2012; Mitra \& Meda, 2009; Ratti, 2001; Sagar \& Kumar, 2010). The selectivity of the drying system for particular products or retention of particular elements in the products is very important for suitable and efficient drying of the products. Each drying technology has its own advantages and disadvantages; one system may work effectively for a product while other systems may not. Furthermore, different drying systems will have different effectiveness for the retention of antioxidant capacities of Saskatoon berries (Kwok, Hu, Durance, \& Kitts, 2004; Mejia-Meza et al., 2010). As such, there is a need to identify an effective, economically viable drying method to retain maximum antioxidant activities of Saskatoon berries.

Antioxidants scavenge free radicals produced in the body during metabolic reactions and prevent the damage of lipids, proteins and DNA caused by free radicals (Mitra, Chang, \& Yoo, 2011). Hence, they are purported to help prevent some diseases such as cancer, inflammation, atherosclerosis, and aging (Jeong et al., 2010; Subhasree, Baskar, Keerthana, Susan, \& Rajasekaran, 2009). Determination of antioxidant activity of fruits and vegetables is therefore important and there are several chemical methods of assessing antioxidant activity. The DPPH (2, 2-Diphenyl-1-picrylhydrazyl) and ABTS (2, 2'Azino-bis (3-ethylbenzothiazoline-6-sulfonic acid) diammonium salt) radical scavenging assays are popular because these methods are simple, inexpensive and widely used to determine total antiox-

\begin{tabular}{l|l|l|l} 
IJFS & October 2013 & Volume 2 & pages 224-237
\end{tabular} 
$226 \mid$ Mitra et al.

idant activities in fruits and vegetables (Floegel, Kim, Chung, Koo, \& Chun, 2011). In the DPPH method, the scavenging of the stable DPPH radical by an antioxidant is measured colorimetrically. The antioxidant reacts with DPPH radical in methanol solution and the reduction of the radical by the antioxidant is determined by monitoring the decrease in absorbance of the solution at $515 \mathrm{~nm}$. The DPPH radical absorbs at $515 \mathrm{~nm}$ but upon reduction by an antioxidant, the absorption disappears (Brand-Williams, Cuvelier, \& Berset, 1995). A 15 or 30 minute reaction time is typically sufficient for measuring the scavenging of the DPPH radicals (Aruoma, 2003). Another colorimetric assay to determine radical scavenging is the ABTS radical scavenging test. The ABTS method is also known as the Trolox Equivalent Antioxidant Capacity (TEAC) assay because the TEAC value is assigned by comparing the scavenging capacity of an antioxidant to that of Trolox, a synthetic, water-soluble analogue of vitamin $\mathrm{E}$ (Re et al., 1999). In this process, the stable ABTS radical is created by reaction with ferrylmyoglobin radical or potassium persulphate. An aliquot of this ABTS radical solution is then mixed with the test antioxidant and allowed to react for a specified reaction time. The ABTS radical scavenging is a fast reaction and the reaction time reported in literature for this test usually varies from only one to six minutes. The ABTS radical has an absorbance maximum at $734 \mathrm{~nm}$ and this absorbance is lost upon reduction of the radical by the antioxidant. The antioxidant suppresses the ABTS radical absorbance to an extent and at a rate dependent on the antioxidant activity. The TEAC value is typically reported as the ability of hydrogen- or electron-donating antioxidant to scavenge the ABTS radical compared to that of Trolox (Re et al., 1999). The TEAC value for Trolox is set at 1.0, thus any antioxidant with a TEAC value greater than 1.0 is considered to be a more effective scavenger of ABTS radical than Trolox, and the greater the TEAC value, the stronger the antioxidant activity (Re et al., 1999). The objective of this research was to compare the retention of antioxidant activities of Saskatoon berries dried with the techniques of freeze drying, microwave-vacuum drying, thin layer hot air drying and vacuum drying.

\section{Materials and Methods}

\subsection{Materials}

Frozen Saskatoon berries (Amelanchier alnifolia Nutt.) were supplied by Riverbend Plantation (Saskatoon, Saskatchewan, Canada). The chemicals 2, 2'-Azino-bis (3-ethylbenzothiazoline6-sulfonic acid) diammonium salt (ABTS), 2, 2Diphenyl-1-picrylhydrazyl (DPPH), 6-Hydroxy2, 5, 7, 8-tetramethylchromane-2-carboxylic acid (Trolox), potassium persulfate, methanol and hydrochloric acid $(\mathrm{HCl})$ were purchased from Sigma-Aldrich Canada Ltd. (Oakville, Ontario, Canada).

\subsection{Drying of Saskatoon Berries}

\section{Thawing of frozen berries}

The frozen Saskatoon berries were kept in frozen storage and prior to drying (except freeze drying), the berries were thawed using a pre-drier to equilibrate with ambient conditions and remove the frozen water from the surface of the berries. The berries were spread on the surface of the pre-drier in a single layer and thawed at approximately $40{ }^{\circ} \mathrm{C}$ for $30 \mathrm{~min}$. The berries were then placed in a perforated ultra-high density polyethylene (UHDP) basket to drain the surface water.

\section{Freeze drying of Saskatoon berries}

Freeze drying of Saskatoon berries was conducted using a FreeZone ${ }^{\circledR} 4.5$ Freeze Dry System (LABCONO, Kansas City, MO, USA). The surface water of frozen Saskatoon berries was removed using a tissue paper and $100 \mathrm{~g}$ of frozen berries were placed into a $300 \mathrm{~mL}$ flask and the flask was connected to the freeze drier though the coldtrap holder of the drier. The berries were dried at -40 ${ }^{\circ} \mathrm{C}$ under high vacuum pressure for about $72 \mathrm{~h}$ to a final moisture content of $12 \% \pm 0.5$. 


\section{Microwave-vacuum drying of Saskatoon berries}

Microwave combined with vacuum drying was performed as per the method followed by Mitra and Meda, 2009. Ten $\mathrm{kg}$ of berries were dried at a microwave power level of $6.0 \mathrm{~kW}$ for $90 \mathrm{~min}$ until the final moisture content of the berries reached $12 \% \pm 0.5$ using a pilot scale microwavevacuum drier (Model MG8KW, Enwave Corporation, Vancouver, Canada). The drier consisted of a cylindrical stainless steel chamber where the microwave field and vacuum was applied. Inside the chamber, berries were contained in a basket made of UHDP that was located on two rollers, also made from UHDP. One of the rollers was connected directly to a variable speed motor through the chamber wall. Cogs in the wall of the basket interlocked with matching $\operatorname{cog}$ s in a roller to provide smooth rotation. Basket rotation speed was set prior to each drying cycle and the rotational speed was kept constant at $10 \mathrm{rpm}$. Vacuum was provided by an external pump and vacuum pressure was kept constant $(100 \mathrm{kPa})$. After completion of microwave-vacuum drying, the dried berries were removed and cooled to room temperature.

\section{Thin layer hot air drying of Saskatoon berries}

A hot air cross flow thin layer drying unit developed by Adapa et al. (Adapa \& Schoenau, 2005; Adapa, Schoenau, \& Sokhansanj, 2002) at the Department of Chemical and Biological Engineering, University of Saskatchewan, Canada, was used to dry the Saskatoon berries using hot air flow. Saskatoon berries (500 g) were placed on a tray within the thin layer drying unit. The berries on the tray were distributed evenly in a single layer on the tray inside the drying unit. The drying air temperature and air flow velocity through the berries were set at $60{ }^{\circ} \mathrm{C}$ and $1.25 \mathrm{~m} \mathrm{~s}^{-1}$, respectively, while the relative humidity inside the drying unit was maintained below 5\%. Throughout the drying process, the temperature, air flow, relative humidity and product mass data were monitored online using a computer with LABVIEW 6.0 (National Instruments, Austin, TX) data acquisition software connected to the drying unit.
The Saskatoon berries were dried for $48 \mathrm{~h}$ to a final moisture content of $12 \% \pm 0.5$.

\section{Vacuum drying of Saskatoon berries}

Vacuum drying of Saskatoon berries was performed using a Fisher Vacuum Oven (Fisher Scientific Company, Ottawa, Canada). Saskatoon berries (500 g) were spread evenly in a thin single layer on the drying tray and placed inside the vacuum oven. The vacuum pressure and the drying temperature were kept constant at $100 \mathrm{kPa}$ and $50{ }^{\circ} \mathrm{C}$, respectively. The berries were dried for 120 $\mathrm{h}$ to a final moisture content of $12 \% \pm 0.5$.

\subsection{Determination of Antioxidant Activity of the Saskatoon Berries}

\section{Extraction of Saskatoon berries}

A $75 \%\left(\mathrm{v} \mathrm{v}^{-1}\right)$ methanol in $1.5 \mathrm{M} \mathrm{HCl}$ solution was prepared to extract the phenolic compounds from the Saskatoon berries. Fifty grams (at 20\% total solid content) of frozen berries or $10 \mathrm{~g}$ of dried berries were mixed with $150 \mathrm{~mL}$ of the $75 \%$ methanol solution and extracted for 5 minutes in a Sunbeam blender (Sunbeam Corporation Canada Ltd, Ontario, Canada). The blended mixture was then transferred into a beaker, covered with a piece of parafilm and stored in a laboratory refrigerator overnight at $4^{\mathrm{o}} \mathrm{C}$. The cooled extract was filtered by vacuum through a Whatman \# 44 filter paper. Sediment on the filter paper was washed with $50 \mathrm{~mL}$ of $75 \%$ methanol and vacuum filtered after washing. The filtrate was transferred into a $250 \mathrm{~mL}$ volumetric flask and brought to volume with further residue washings. All extractions and analyses were done in duplicate. The extracted sample was kept in a refrigerator for 2 days until analysis for antioxidant activity and total anthocyanin content.

\section{DPPH Radical Scavenging Assay}

A $500 \mu \mathrm{M}$ DPPH solution was prepared by adding $9.8 \mathrm{mg}$ of $\mathrm{DPPH}$ to a $50 \mathrm{~mL}$ volumetric flask and diluting to volume with $70 \%\left(\mathrm{v} \mathrm{v}^{-1}\right)$ aqueous methanol. In order to ensure that all solu- 
ble components were dissolved the DPPH solution was placed in an FS ultrasonic bath $\left(20 \pm 3^{\mathrm{O}} \mathrm{C}\right.$; Fisher Scientific, Edmonton, AB) for 20 minutes. Fresh solutions were prepared on each day of analysis. Three sample solutions of crude, 5 fold and 10 fold extract dilutions with $70 \%$ methanol in 1.5 $\mathrm{M} \mathrm{HCl}$ were prepared to produce DPPH radical scavenging levels ranging from approximately 10 to $85 \%$. A $0.25 \mathrm{~mL}$ aliquot of the sample solution was added to $2 \mathrm{~mL}$ of DPPH in a $20 \mathrm{~mL}$ test tube. A control solution (blank) was prepared by adding $0.25 \mathrm{~mL} 70 \%\left(\mathrm{v} \mathrm{v}^{-1}\right)$ methanol to $2 \mathrm{~mL}$ DPPH solution. Samples were vortexed on level 5 for 15 seconds (Vortex-Genie 2, Scientific Industries, Inc, USA) and held at room temperature $\left(22 \pm 3^{\mathrm{O}} \mathrm{C}\right)$ in the dark for 15 minutes. The absorbance of the sample and control solutions was determined at $517 \mathrm{~nm}$ with a spectrophotometer (Jenway Genova, Jenway Ltd., UK) and the percent DPPH radical scavenging (\% inhibition) was calculated using equation (1). In equation (1), $\mathrm{A}_{517}$ sample and $\mathrm{A}_{517}$ blank denote absorbance at $517 \mathrm{~nm}$ for sample and blank (control), respectively.

$$
\% \text { DPPH inhibition }=\left[1-\left(\frac{A 517 \text { sample }}{\text { A517 blank }}\right)\right] \times 100
$$

A plot of the \% inhibition (\% DPPH radical scavenging) versus concentration of each sample was constructed. The average correlation coefficient of a linear regression equation for each drying system was $\geq 0.99$. The $\%$ inhibition of $1 \mathrm{mM}$ Trolox solution was measured and the concentration of the sample that resulted in the same level of DPPH radical scavenging as the Trolox was determined from the regression equation. The DPPH radical scavenging activity of the fruit sample was expressed as mM of Trolox $100 \mathrm{~g}^{-1}$ dry fruit (dwb). All samples and standards were analyzed in duplicate.

\section{ABTS radical Scavenging Activity (TEAC assay)}

A $7 \mathrm{mM}$ ABTS stock solution was prepared by adding $19 \mathrm{mg}$ of ABTS into $5 \mathrm{~mL}$ of deionized water $\left(3.8 \mathrm{mg} \mathrm{mL}^{-1}\right)$. A $7 \mathrm{mM}$ potassium persulfate solution was prepared by dissolving 18.9 $\mathrm{mg}$ of potassium persulfate into $10 \mathrm{~mL}$ of deionized water. In order to produce ABTS radical cations (ABTS•+), $4 \mathrm{~mL}$ of the ABTS stock solution was added to $2 \mathrm{~mL}$ of $7 \mathrm{mM}$ potassium persulfate solution and the mixture was held in the dark for 12 to $16 \mathrm{~h}$ at ambient temperature to ensure complete oxidation of ABTS prior to analysis. The generation of ABTS radical using potassium persulfate was performed according to the method described by Re et al. (1999). The resulting ABTS•+ solution was diluted with aqueous $70 \%$ methanol in the ratio of 1:55 (ABTS•+: aqueous $70 \%$ methanol) to produce a spectrophotometric absorbance reading of 0.75 to 0.9 at 734 $\mathrm{nm}$. Three diluted sample solutions were similar to DPPH radical scavenging assay. Twenty microlitres of diluted sample solution was added to $2 \mathrm{~mL}$ of ABTS•+ solution in a $20 \mathrm{~mL}$ test tube. A blank solution was prepared by adding $20 \mu \mathrm{L}$ of acidified $70 \%$ methanol to $2.0 \mathrm{~mL}$ of ABTS•+ solution. Samples were vortexed on level 5 for 15 seconds (Vortex-Genie 2, Scientific Industries, Inc, USA) and the absorbance of the sample and control solutions was determined at $734 \mathrm{~nm}$ with a spectrophotometer (Jenway Genova, Jenway Ltd., $\mathrm{UK})$ after $1 \mathrm{~min}$ reaction. The $\%$ inhibition of the ABTS radical was calculated using equation (2). A $1 \mathrm{mM}$ Trolox solution was prepared by adding $25.03 \mathrm{mg}$ of Trolox to $100 \mathrm{~mL}$ of acidified $75 \%$ methanol. The \% inhibition of the $1 \mathrm{mM}$ Trolox solution was determined using the same technique as for the sample solutions. The \% ABTS•+ inhibition was plotted as a function of sample concentration and the linear regression equations were generated. Correlation coefficients of the linear regression equations were $\geq 0.98$. The $\%$ ABTS• + inhibition of $1 \mathrm{mM}$ Trolox was determined from the slope of the Trolox linear regression curve $(r=0.99)$ and the sample concentration equivalent to the inhibition activity of $1 \mathrm{mM}$ Trolox was calculated using equation (3). Trolox equivalent antioxidant capacity (TEAC) was expressed as the equivalent activity of Trolox (mM) in $100 \mathrm{~g}$ of sample and was calculated using equation (4). The analysis was performed in duplicate for all samples.

$\%$ ABTS inhibition $=\left(1-\frac{\text { A734 sample }}{\text { A734 ABTS solution }}\right) \times 100$

\begin{tabular}{l|l|l|l} 
IJFS & October 2013 & Volume 2 & pages 224-237
\end{tabular} 


$$
\begin{gathered}
C_{T E}=B+(S \times Y) \\
\mathrm{TEAC}=100 / C_{T E}
\end{gathered}
$$

where,

$\mathbf{A}_{734} \mathbf{n m}$ sample $=$ absorbance of the sample @ $734 \mathrm{~nm}$ after one min reaction;

$\mathbf{A}_{734} \mathbf{n m}$ blank = absorbance of the blank @ $734 \mathrm{~nm}$ after one min reaction;

$\mathbf{C}_{T E}=$ sample concentration $\left(\mathrm{mg} \mathrm{mL}^{-1}\right)$ producing ABTS•+ inhibition equivalent to 1 mM Trolox;

$\mathbf{B}=$ intercept of the sample linear regression equation;

$\mathbf{S}=$ slope of the sample linear regression equation;

$\mathbf{Y}=\%$ ABTS $\bullet+$ inhibition of $1 \mathrm{mM}$ Trolox under assay conditions (the average value of $\mathrm{Y}$ for this study was 39.5 );

TEAC = Trolox equivalent antioxidant capacity (Trolox equivalents in $100 \mathrm{~g}$ sample).

\subsection{Determination of total anthocyanin of the extracts}

Total anthocyanin content of Saskatoon berry extract was determined according to the $\mathrm{pH}$ differential method described by Fuleki and Francis (1968) and Wrolstad (1993). A pH 1.0 buffer solution was prepared by mixing $125 \mathrm{~mL}$ of 0.2 $\mathrm{N} \mathrm{KCl}$ with $385 \mathrm{~mL}$ of $0.2 \mathrm{~N} \mathrm{HCl}$ and $490 \mathrm{~mL}$ deionized water. The $\mathrm{pH}$ of the buffer was adjusted to $\mathrm{pH} 1.0$ with $0.2 \mathrm{~N} \mathrm{HCl}$. A pH 4.5 buffer solution was prepared by mixing $440 \mathrm{~mL} 1.0 \mathrm{M}$ sodium acetate with $200 \mathrm{~mL} 1.0 \mathrm{M} \mathrm{HCl}$ and 360 $\mathrm{mL}$ deionized water. The $\mathrm{pH}$ of the solution was measured and adjusted to $\mathrm{pH} 4.5$ with $1.0 \mathrm{M} \mathrm{HCl}$. One milliliter of anthocyanin extract was diluted to $25 \mathrm{~mL}$ in each of the $\mathrm{pH} 1.0$ and 4.5 buffers and allowed to equilibrate in the dark for two hours. The absorbance of the samples at $512 \mathrm{~nm}\left(\mathrm{~A}_{512}\right.$ $\mathrm{nm})$ and $700 \mathrm{~nm}\left(\mathrm{~A}_{700} \mathrm{~nm}\right)$ was measured with a spectrophotometer (Jenway Genova, Jenway Ltd., $\mathrm{UK})$. The difference in absorbance $(\Delta \mathrm{A})$ between the anthocyanin extract diluted in $\mathrm{pH} 1.0$ and $\mathrm{pH}$ 4.5 buffers was calculated using equation (5). The $\mathrm{A}_{700} \mathrm{~nm}$ was considered in the calculation of $\Delta \mathrm{A}$ to correct for any background absorbance due to turbidity in the samples. The anthocyanin content was expressed as mg cyanidin 3-glucoside 100 $\mathrm{g}^{-1}$ berries using a molar absorbance coefficient $(\in)$ of $26900 \mathrm{~L}^{-1} \mathrm{M}^{-1} \mathrm{~cm}^{-1}$ (Wrolstad, 1993) and was calculated using equation (6) where: MW is the molecular weight of cyanidin 3-glucoside (449.2 $\mathrm{g} \mathrm{L}^{-1}$ ) and DF is the dilution factor to express the samples on a per gram of fruit basis. All samples were analyzed in duplicate.

$\Delta A=\left(A_{512} \mathrm{~nm} \mathrm{pH} 1.0-A_{700} \mathrm{~nm} \mathrm{pH} 1.0\right)-\left(A_{512} \mathrm{~nm} \mathrm{pH} 4.5-A_{700} \mathrm{~nm} \mathrm{pH} 4.5\right)$

Total anthocyanin content $=\frac{A \Delta \times M W \times D F \times 100}{\in} \times 100$ 


\subsection{Statistical analysis}

All measurements were conducted in duplicate and the results are shown as mean \pm standard deviation (SD). One way (single factor) ANOVA was performed using Microsoft Excel 2010 to determine the significant effects among the drying treatments at $\mathrm{P}<0.05$. In order to determine the differences among the results of the mean of the drying treatments, Duncan Multiple Range Test (DMRT) was performed at $\mathrm{P}<0.05$ using statistical software SPSS 14.0 for Windows.

\section{Results and Discussion}

\subsection{Drying effect on DPPH radical scavenging of berries}

The DPPH radical scavenging activity of the frozen Saskatoon berries and freeze, microwavevacuum, thin layer hot air and vacuum dried Saskatoon berries was determined and expressed as mM Trolox equivalents per $100 \mathrm{~g}$ dry fruit. Frozen Saskatoon berries contained a moisture content of $82 \% \pm 2$ and the dried berries for all the drying treatment contained the moisture content of $12 \% \pm 0.5$. The antioxidant activities of the samples are presented in Table 1. ANOVA analysis between the samples is summarized in Table 2. The frozen sample (control) showed the highest antioxidant activity of $28.25 \pm 0.35$ ( $\mathrm{mM}$ Trolox $100 \mathrm{~g}^{-1}$ ) among the all samples tested. The observations from the DPPH radical scavenging activity (Table 1) showed that drying affected the retention of antioxidant activity of the dried berries and reduced antioxidant activities of the berries for the all drying treatments. However, the degree of loss of antioxidant activity of the dried berries varied with the drying technique. The freeze dried sample showed the highest antioxidant activity of $18.55 \pm 0.35$ (mM Trolox $100 \mathrm{~g}^{-1}$ ) and the vacuum dried sample showed the lowest antioxidant activity of $4.55 \pm$ 0.78 (mM Trolox $100 \mathrm{~g}^{-1}$ ) among the all dried samples. Microwave-vacuum dried sample followed the freeze dried sample and thin layer hot air dried sample followed the microwave-vacuum dried sample in terms of higher antioxidant retention rank among the samples tested. The one-way single factor ANOVA (Table 2) showed the F value (913.72) was greater than the $\mathrm{F}$ value at critical point (Fcrit) (5.19). Since the F value exceeded the Fcrit the null hypothesis was rejected. This result indicated that the sample means tested were different and the group means were not equal (Lurie, Abramson, \& Vail, 2011). Also, the P value $<0.05$ indicated a significant difference between the samples tested. The lower the $\mathrm{P}$ value designated the more significant difference between the groups (Lurie et al., 2011). Since ANOVA analysis cannot determine whether each individual mean is significantly different from all of the other means of the group, a Duncan's Multiple Range Test (DMRT) was performed. The DMRT analysis showed that the DPPH radical scavenging of each sample was significantly different for all samples (Table 1). The ranking of DPPH radical scavenging activity of the dried Saskatoon berries in descending order was freeze drying $>$ microwavevacuum drying $>$ thin layer hot air drying $>$ vacuum drying (Table 1). This finding was in agreement with that of Kwok et al. (2004) for dried Saskatoon berries and Mejia-Meza et al. (2010) for dried raspberries. The freeze drying process involves drying of frozen berries by sublimation. The low temperature and solid state of water during freeze drying protect primary structure of the berries and retain the color of the berries. Ratti (2001) reported a close relationship between antioxidant content, vitamin content and color of the products. In addition, due to the absence of liquid water during the freeze drying there was minimal loss of anthocyanins and other polyphenols; hence, this drying technique retained the highest antioxidant activities in dried Saskatoon berries. Microwave drying involves volumetric heating whereby the berries absorb the microwave energy directly and convert it into heat internally, and the generated heat transfers from inside to the surface of the berries. The vacuum helps to dry the berries under a low temperature. The combination of microwave and vacuum drying improved the color attribute and reduced the loss of anthocyanins and polyphenols because of the low temperature and short period of drying time, and the 
Table 1: Experimental results for total anthocyanin content, DPPH radical scavenging and TEAC (ABTS radical scavenging) for frozen and differently dried Saskatoon berry samples

\begin{tabular}{lccc}
\hline \multicolumn{1}{c}{ Sample } & $\begin{array}{c}\text { DPPH radical scavenging } \\
\left(\mathrm{mM} \mathrm{TE} 100 \mathrm{~g}^{-1}, \mathrm{dwb}\right)\end{array}$ & $\begin{array}{c}\text { TEAC } \\
\left(\mathrm{mM} \mathrm{TE} 100 \mathrm{~g}^{-1}, \mathrm{dwb}\right)\end{array}$ & $\begin{array}{c}\text { Total anthocyanin } \\
\left(\mathrm{mg} 100 \mathrm{~g}^{-1}, \mathrm{dwb}^{2}\right.\end{array}$ \\
\hline Frozen Saskatoon & $28.25^{a} \pm 0.35$ & $5.45^{a} \pm 1.06$ & $988^{a} \pm 20$ \\
Freeze Drying & $18.55^{b} \pm 0.35$ & $3.90^{b} \pm 0.00$ & $607^{b} \pm 18$ \\
Microwave-vacuum drying & $12.70^{c} \pm 0.00$ & $3.60^{b c} \pm 0.71$ & $279^{c} \pm 20$ \\
Hot air drying & $9.85^{d} \pm 0.21$ & $3.35^{b c} \pm 0.21$ & $203^{d} \pm 25$ \\
Vacuum drying & $4.55^{e} \pm 0.78$ & $2.25^{c} \pm 0.21$ & $129^{e} \pm 10$ \\
\hline
\end{tabular}

$\mathrm{TE}=$ Trolox equivalent, Means within a column with different letters are significantly different $(\mathrm{P}<0.05)$.

Table 2: ANOVA for total anthocyanin content, DPPH radical scavenging and TEAC (ABTS radical scavenging) of frozen and differently dried Saskatoon berry samples

\begin{tabular}{lcccccc}
\hline Source & $S S$ & $d f$ & $M S$ & $F$ & P-value & $F$ crit \\
\hline $\begin{array}{l}\text { DPPH radical scavenging } \\
\left(\mathrm{mM} \mathrm{TE} 100 \mathrm{~g}^{-1} \text {, dwb) }\right.\end{array}$ & 657.88 & 4 & 164.47 & 913.72 & $<0.05$ & 5.19 \\
TEAC & 10.67 & 4 & 2.67 & 7.78 & 0.02 & 5.19 \\
$\begin{array}{l}\left.\text { (mM TE } 100 \mathrm{~g}^{-1}, \mathrm{dwb}\right) \\
\text { Total Anthocyanin content }\end{array}$ & 1014463 & 4 & 253615.9 & 958.13 & $<0.05$ & 5.19 \\
$\left(\mathrm{mg} 100 \mathrm{~g}^{-1}, \mathrm{dwb}\right)$ & & & & & & \\
\hline
\end{tabular}

absence of air during drying protected the fruit from oxidation.

In the thin layer hot air drying, the berries were exposed under a continuous hot air stream flow for a relatively long time to increase the temperature to evaporate moisture from the fruit (Ratti, 2001). The high temperature, long drying time and presence of air led to oxidative degradation of the anthocyanins (Table 1), resulting in the deterioration of the color attribute of the dried fruit (Dev \& Raghavan, 2012; Ratti, 2001). Thus thin layer hot air drying showed less antioxidant retention capacity than microwave-vacuum drying. Vacuum drying alone resulted in a greater loss of antioxidant activity of the Saskatoon berries. This result was probably due to, in spite of low temperature, the long drying time which allowed for considerable leaching loss of anthocyanins as well as oxidation of the anthocyanins in the Saskatoon berries.

The effect of concentration $\left(\mathrm{mg} \mathrm{mL}^{-1}\right)$ of the extract on the inhibition of DPPH radical (\%) is shown in Figure 1. The results show that the $\%$ inhibition of DPPH radical increased with the increase of the concentration $\left(\mathrm{mg} \mathrm{mL}^{-1}\right)$ of the extracts. A linear correlation between DPPH radical scavenging and concentration of the extract was found for vacuum drying and microwavevacuum drying with $\mathrm{R}^{2}$ values of 0.99 and 0.91 , respectively. Frozen berries, and berries dried by freeze drying and thin layer hot air drying did not show a linear relationship between the extract concentration and DPPH radical scavenging. However, there was a significant difference between different tested samples at $\mathrm{P}$ value $<0.05$. It is anticipated that vacuum may influence the drying mechanism to have a linear relationship between concentration of the extracts and the DPPH radical scavenging for vacuum drying and microwave-vacuum drying of Saskatoon berries.

\subsection{Drying effect on ABTS radical scavenging (TEAC assay) of the berries}

Trolox equivalent antioxidant capacity (TEAC) of the frozen, freeze dried, microwave-vacuum dried, thin layer hot air dried and vacuum dried Saskatoon berries is shown in Table 1. All the dried samples (moisture content $12 \% \pm 0.5$ ) showed lower TEAC values compared to the frozen sample. This trend was similar to DPPH radical scavenging in terms of antioxidant activity re- 
232 | Mitra et al.

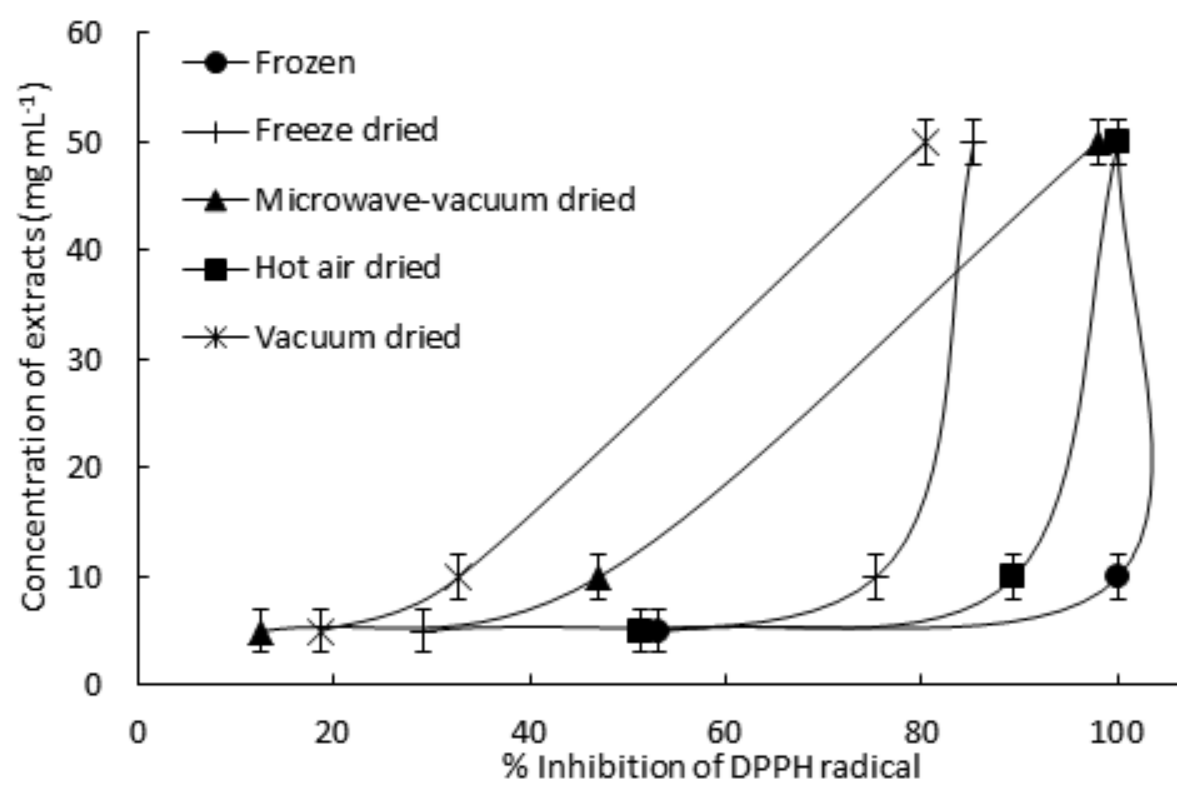

Figure 1: Effect of concentrations $\left(\mathrm{mg} \mathrm{mL}^{-1}\right)$ of Saskatoon berry extracts on the \% inhibition of DPPH radical for the drying systems tested

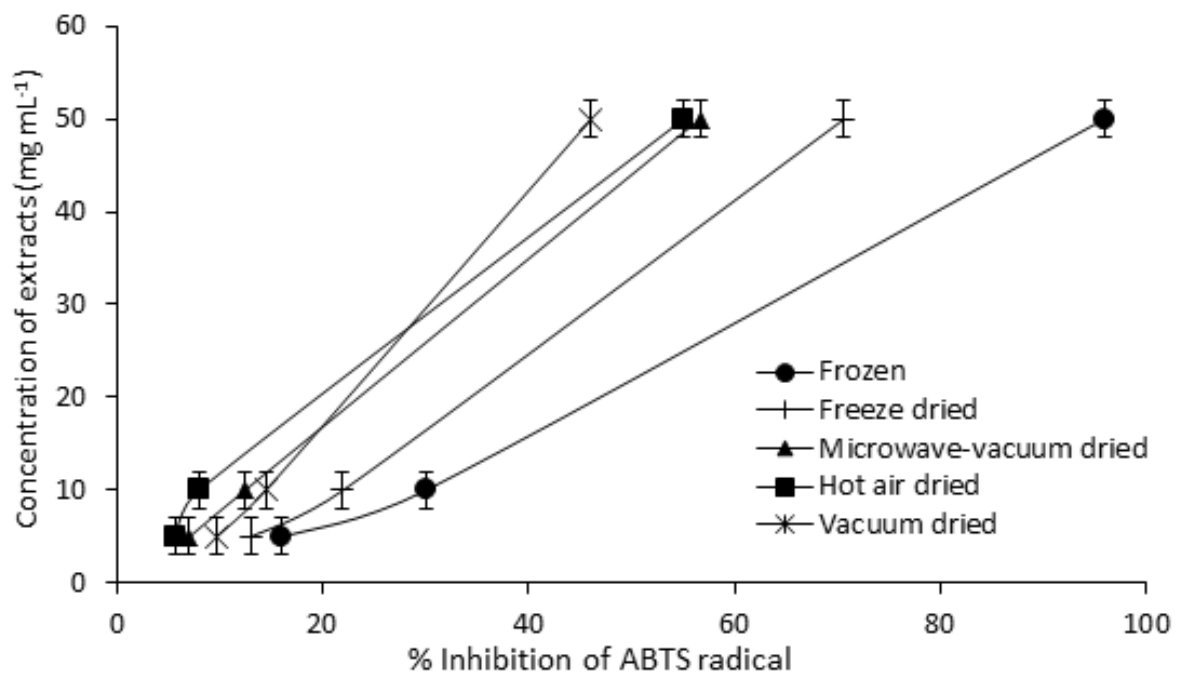

Figure 2: Effect of concentrations $\left(\mathrm{mg}^{\left.-\mathrm{mL}^{-1}\right)}\right.$ of Saskatoon berry extracts on the \% inhibition of ABTS radical for the drying systems tested 
tention of the dried berries. The frozen Saskatoon berries exhibited the highest antioxidant activity $5.45 \pm 1.06$ (mM Trolox $100 \mathrm{~g}^{-1}$ ) and vacuum dried berries showed the lowest antioxidant activity among the samples tested. The observed antioxidant activity ranking of the fruit in terms of TEAC (ABTS radical scavenging) was frozen berries $>$ freeze dried berries $>$ microwavevacuum dried berries $>$ thin layer hot air dried berries $>$ vacuum dried berries. In the freeze drying process, the low temperature and solid state of water in the material during drying resulted in improved retention capacity of nutrients of the products (Dev \& Raghavan, 2012; Ratti, 2001). Our results indicate that less thermal degradation and less leaching loss during freeze drying helped to retain the highest antioxidant activity $(3.90 \pm 0.01$, mM Trolox $100 \mathrm{~g}^{-1}$ ) among the dried samples. Microwave-vacuum drying with an antioxidant activity of $3.60 \pm 0.71\left(\mathrm{mM}\right.$ Trolox $\left.100 \mathrm{~g}^{-1}\right)$ followed freeze drying. Kwok et al. (2004)) showed that microwave-vacuum dried Saskatoon berries exhibited higher total anthocyanin and polyphenol levels, which were associated with a greater antioxidant activity compared to hot air dried berries.

The results of ANOVA between the samples for TEAC are presented in Table 2. The $\mathrm{F}$ value 7.78 exceeded the $\mathrm{F}$ value (5.19) at critical point (Fcrit) and $\mathrm{P}$ value $(0.02)<0.05$ (Table 2). These indicate that the means of the samples tested were significantly different (Lurie et al., 2011). Duncan's Multiple Range Test (DMRT) was conducted to identify the significant difference between each sample. The DMRT analysis ranked the samples (most significant to least significant) as frozen berries $>$ freeze dried berries, microwave-vacuum dried berries and thin layer hot air dried berries > microwave-vacuum dried berries, thin layer hot air dried berries and vacuum dried berries. The means between freeze drying and microwave and thin layer hot air drying and between microwave-vacuum drying and thin layer hot air drying and vacuum drying were not significantly different using the DMRT statistical analysis (Table 1).

The relationship between the concentration (mg $\mathrm{mL}^{-1}$ ) of the extract and \% inhibition of ABTS radical is shown in Figure 2. There was a significant difference between different tested samples at $\mathrm{P}$ value $<0.05$. The results showed that the
$\%$ inhibition of ABTS radical increased with the increase of the concentration $\left(\mathrm{mg} \mathrm{mL}^{-1}\right)$ of the extracts. The ABTS radical scavenging was linearly correlated to the concentration of the extract with $\mathrm{R}^{2}$ values of $0.996,0.998,0.999,0.996$ and 0.999 , respectively, for frozen, freeze drying, microwave-vacuum drying, thin layer hot air drying and vacuum drying.

\subsection{Drying effect on total anthocyanin content of berries}

The total anthocyanin concentration for frozen Saskatoon berries, freeze dried berries, microwave-vacuum dried berries, thin layer hot air dried berries and vacuum dried berries was determined to investigate the effect of drying techniques on the retention of these phenolic pigments. All the dried samples contained a moisture content of $12 \% \pm 0.5$. The results of total anthocyanin content in the samples are shown in Table 1. Frozen Saskatoon berries contained the highest anthocyanin content of $988 \pm 20.00 \mathrm{mg} 100 \mathrm{~g}^{-1}$ dry fruit and vacuum drying showed the poorest anthocyanin retention $\left(129 \pm 1.00 \mathrm{mg} 100 \mathrm{~g}^{-1}\right.$ dry fruit) among the samples. Freeze drying showed the highest potential for retaining anthocyanin content in the samples tested in comparison to the other drying techniques. The anthocyanin content of microwave-vacuum dried fruit followed the freeze drying and thin layer hot air drying followed microwave-vacuum drying. Drying at higher temperatures can lead to loss of anthocyanins because these pigments are heat sensitive and are unstable during thermal treatment (Idham, Muhamad, Setapar, \& Sarmidi, 2012; Patras, Brunton, O'Donnell, \& Tiwari, 2010). As the intensity and duration of the heat treatment increases, the anthocyanins become more unstable and susceptible to degradation. Patras et al. (2010) reported that an arithmetic increase in temperature results in a logarithmic increase in anthocyanin destruction. The presence of oxygen can also accelerate the destruction of anthocyanin by direct chemical oxidation or through the effects of oxidative enzymes (Kwok et al., 2004; Patras et al., 2010). In our study, the frozen Saskatoon berries retained the highest concentration of anthocyanins attributable to no thermal influence 
or oxidative damage. Microwave-vacuum drying showed better retention of anthocyanin in the dried product than thin layer hot air drying. Microwave with the combination of vacuum helped to retain higher anthocyanin in dried berries because of lower temperature, shorter drying time and less exposure to air. On the other hand, thin layer hot air dried berries processed with a high temperature and longer drying time and exposure to air resulted in a lower anthocyanin content in the dried berries. Vacuum drying alone resulted in poor retention of anthocyanin content in the vacuum dried berries, attributable to the relatively long drying time $(120 \mathrm{~h})$.

ANOVA and Duncan's Multiple Range Test (DMRT) were conducted to determine the statistical evidence between the samples for total anthocyanin and confirm the results statistically. The ANOVA results (Table 2) showed that there was a significant difference between the anthocyanin content of the samples tested, as the $\mathrm{F}$ value $(958.13)>$ F crit $(5.19)$ and $\mathrm{P}$ value $<0.05$ (Lurie et al., 2011). The DMRT analysis (Table 1 for anthocyanin) provided clear difference from one sample to others showing frozen $>$ freeze drying $>$ microwave-vacuum drying $>$ thin layer hot air drying $>$ vacuum drying.

\subsection{Correlation between anthocyanin and antioxidant activity}

Regression analysis was performed to determine the correlation between the reduction of anthocyanin content in different dried samples and the reduction of corresponding antioxidant activities determined by DPPH radical scavenging and ABTS radical scavenging and, also, between DPPH radical scavenging and ABTS radical scavenging. The correlation between the reduction of anthocyanin content (\%) for the dried samples and their corresponding reduction in percentage of antioxidant activity determined by DPPH scavenging and TEAC (ABTS scavenging) is shown in Figure 3. The sample of frozen Saskatoon berries was considered as the control. The percent reduction of anthocyanin content and the corresponding antioxidant activity for freeze dried berries, microwave-vacuum dried berries, thin layer hot air dried berries and vacuum dried berries were compared with the frozen (control) berry sample. The observed reductions of anthocyanin contents from the control sample were $39 \%, 72 \%, 80 \%$ and $87 \%$ for freeze dried berries, microwave vacuum dried berries, thin layer hot air dried berries and vacuum dried berries, respectively. The antioxidant activity reductions determined by DPPH radical scavenging were $34 \%, 55 \%, 65 \%$ and $84 \%$, and the antioxidant reductions determined by ABTS radical scavenging were $29 \%, 34 \%, 39 \%$ and $59 \%$ for the freeze dried berries, microwave vacuum dried berries, thin layer hot air dried berries and vacuum dried berries, respectively. Regression analysis showed that the reduction of anthocyanin content in dried berries was linearly correlated with the reduction of DPPH radical scavenging with an $\mathrm{R}^{2}$ value of 0.97 at $\mathrm{P}<0.05$ and, also, linearly correlated with the reduction of ABTS radical scavenging with an $\mathrm{R}^{2}$ value of 0.88 at $\mathrm{P}<$ 0.05 . The results showed that antioxidant activity of the berries was significantly attributable to the presence of total anthocyanin content of the berry samples. This result is in the agreement with Mazza (1986) that the major anthocyanins found in Saskatoon berries were primarily accountable for the antioxidant activity of the berries. $\mathrm{Hu}$ et al. (2005) also found a strong correlation between antioxidant activities of the Saskatoon berries and anthocyanin content of the berries. Anthocyanins have also been reported to be the principle constituents responsible for the antioxidant activity of raspberries (Mejia-Meza et al., 2010). However, in addition to the significant effect of anthocyanin concentration, other polyphenol compounds may also contribute to the antioxidant activity of the Saskatoon berries (Hu et al., 2005; Mazza, 1986) and it is anticipated that the dehydration techniques affected those compounds during the drying period.

The regression analysis also showed that the DPPH radical scavenging and ABTS radical scavenging activities were correlated linearly with an $\mathrm{R}^{2}$ value of 0.99 at $\mathrm{P}<0.05$. Both of these assays showed a similar trend of determining antioxidant activity of the samples tested, showing their effectiveness for the determination of the antioxidant activity of the Saskatoon berries. However, the DPPH radical scavenging assay was more sensitive than the ABTS radical scavenging

IJFS | October 2013 | Volume 2 pages 224-237 


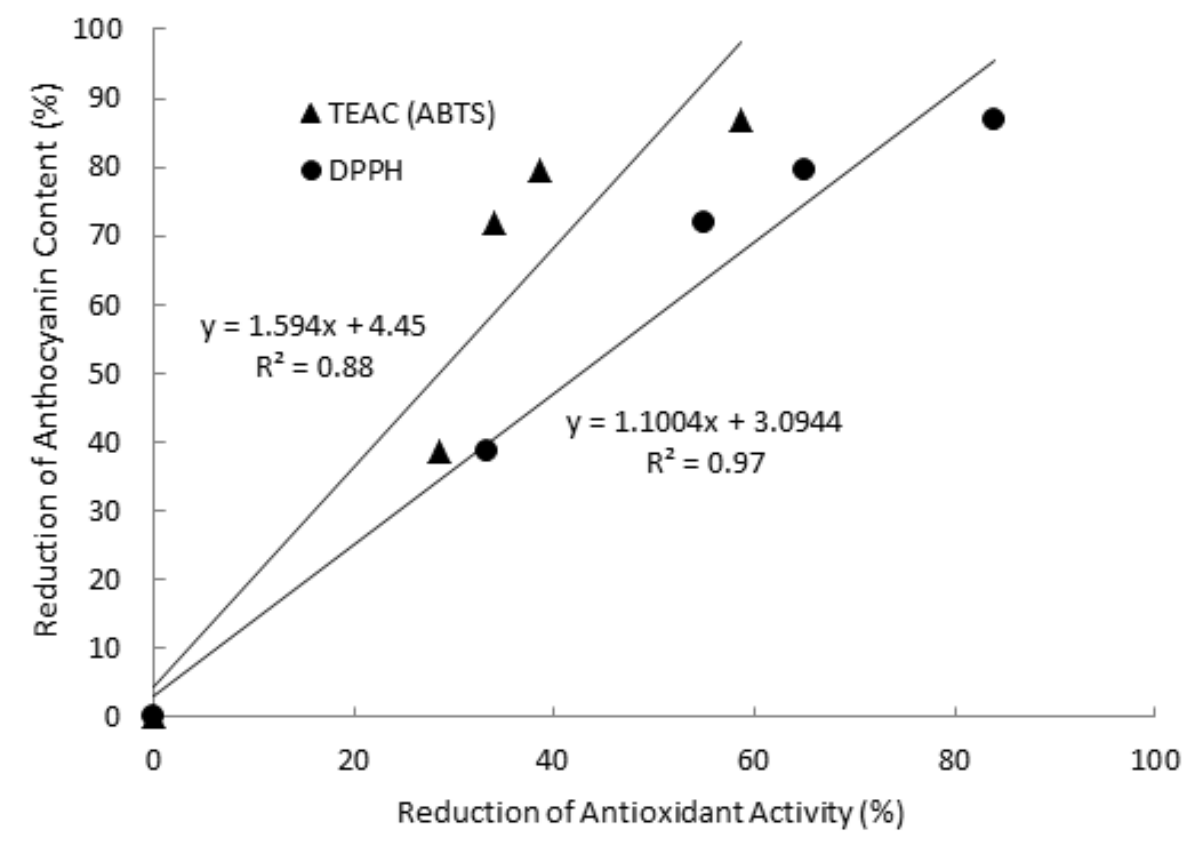

Figure 3: Correlation between the reduction of anthocyanin content (\%) and antioxidant activity (\%) for Trolox equivalent DPPH radical scavenging $(\bigcirc)$ and TEAC (ABTS radical scavenging) $(\Delta)$ for the drying systems tested

assay for determining antioxidant activity of the frozen and dried Saskatoon berries. These results are in agreement with those reported by GramzaMichalowska and Czlapka-Matyasik (2011) who observed that the Trolox equivalent antioxidant activity determined by DPPH radical scavenging was three times higher than the Trolox equivalent antioxidant activity determined by ABTS radical scavenging activity in extract from carrots.

\section{Conclusions}

The different drying techniques used in this study resulted in variability in the retention of anthocyanin and antioxidant activity in the Saskatoon berry extracts. There was a significant difference at $\mathrm{P}<0.05$ between the drying techniques and the results showed that the retention rank (from higher to lower) of anthocyanin and antioxidant activity was freeze drying $>$ microwave-vacuum drying $>$ thin layer hot air drying $>$ vacuum drying. There was a linear correlation between
DPPH radical scavenging and ABTS radical scavenging of the fruit extracts. The DPPH radical scavenging showed greater sensitivity than ABTS radical scavenging and the anthocyanin content of dried berry samples was linearly correlated with both the DPPH radical scavenging and ABTS radical scavenging activity. This result suggested that the antioxidant activity of the frozen and dried berries was largely influenced by the anthocyanin concentration of the fruit. It is expected that the findings of this research will be useful in designing a commercial Saskatoon berry drying system.

\section{Acknowledgements}

We gratefully acknowledge NSERC and Agricultural Development Fund (ADF), SK, Canada for financial support and Grace Whittington, owner of the Riverbend Plantation, for providing the Saskatoon berries.

IJFS | October 2013 | Volume 2 |pages 224-237 


\section{References}

Adapa, P., \& Schoenau, G. (2005). Re-circulating heat pump assisted continuous bed drying and energy analysis. International Journal of Energy Research, 29(11), 961-972. doi:10. 1002/er.1103

Adapa, P., Schoenau, G., \& Sokhansanj, S. (2002). Performance study of a heat pump dryer system for specialty crops - part 1: development of a simulation model. International Journal of Energy Research, 26(11), 10011019. doi:10.1002/er.836

Aruoma, O. I. (2003). Methodological considerations for characterizing potential antioxidant actions of bioactive components in plant foods. Mutation Research/Fundamental and Molecular Mechanisms of Mutagenesis, 523-524(0), 9 -20. doi:10.1016/S0027-5107(02)00317-2

Brand-Williams, W., Cuvelier, M., \& Berset, C. (1995). Use of a free radical method to evaluate antioxidant activity. $\{L W T\}$ Food Science and Technology, 28(1), 25 30. doi:10.1016/S0023-6438(95)80008-5

Dev, S. R. S., \& Raghavan, V. G. S. (2012). Advancements in drying techniques for food, fiber, and fuel. Drying Technology, 30(1112, SI), 1147-1159. doi:10.1080/07373937. 2012.692747

Floegel, A., Kim, D.-O., Chung, S.-J., Koo, S. I., \& Chun, O. K. (2011). Comparison of abts/dpph assays to measure antioxidant capacity in popular antioxidant-rich us foods. Journal of Food Composition and Analysis, 24 (7), 1043-1048. doi:10.1016/j. jfca.2011.01.008

Fuleki, T, \& Francis, F. (1968). Quantitative methods for anthocyanins .1. extraction and determination of total anthocyanin in cranberries. Journal Of Food Science, 33(1), 72 \&. doi:10.1111/j.1365-2621.1968.tb00887.x

Gramza-Michalowska, A., \& Czlapka-Matyasik, M. (2011). Evaluation of the antiradical potential of fruit and vegetable snacks. Acta Sci. Pol., Technol. Aliment. 10(1), 61-72.

$\mathrm{Hu}$, C, Kwok, B., \& Kitts, D. (2005). Saskatoon berries (amelanchier alnifolia nutt.) scavenge free radicals and inhibit intracellular oxidation. Food Research Interna- tional, 38(8-9), 1079-1085. 3rd International Congress on Pigments in Food, Quimper, FRANCE, JUN 14-17, 2004. doi:10 . 1016/j.foodres.2005.02.024

Idham, Z., Muhamad, I. I., Setapar, S. H. M., \& Sarmidi, M. R. (2012). Effect of thermal processes on roselle anthocyanins encapsulated in different polymer matrices. Journal of Food Processing and Preservation, 36(2), 176-184. doi:10.1111/j. 1745-4549. 2011. 00572.x

Jeong, J.-H., Jung, H., Lee, S.-R., Lee, H.J., Hwang, K. T., \& Kim, T.-Y. (2010). Anti-oxidant, anti-proliferative and antiinflammatory activities of the extracts from black raspberry fruits and wine. Food Chemistry, 123(2), 338-344. doi:10 . 1016 / j . foodchem.2010.04.040

Kwok, B. H. L., Hu, C., Durance, T., \& Kitts, D. D. (2004). Dehydration techniques affect phytochemical contents and free radical scavenging activities of saskatoon berries (amelanchier alnifolia nutt.) Journal of Food Science, 69(3), SNQ122-SNQ126. doi:10 . 1111/j.1365-2621.2004.tb13381.x

Lavola, A., Karjalainen, R., \& Julkunen-Tiitto, R. (2012). Bioactive polyphenols in leaves, stems, and berries of saskatoon (amelanchier alnifolia nutt.) cultivars. Journal of Agricultural and Food Chemistry, 60(4), 1020-1027. doi:10.1021/jf204056s

Lurie, D., Abramson, L., \& Vail, J. (2011). Applying statistics (nureg-1475, revision 1 ed.): U.S. Nuclear Regulatory Commission, Washington, $D C$.

Mazza, G. (1986). Anthocyanins and other phenolic-compounds pf saskatoon berries (amelanchier-alnifolia nutt). Journal of Food Science, 51(5), 1260-1264. doi:10 . 1111/j.1365-2621.1986.tb13100.x

Mazza, G. (1982). Chemical-composition of saskatoon berries (amelanchier-alnifolia nutt). Journal of Food Science, 47(5), 1730-1731. doi:10.1111/j.1365-2621.1982.tb05022.x

Mazza, G., \& Cottrell, T. (2008). Carotenoids and cyanogenic glucosides in saskatoon berries (amelanchier alnifolia nutt.) Journal of Food Composition and Analysis, 21 (3), 249-254. doi:10.1016/jjfca.2007.11.003 
Mejia-Meza, E. I., Yanez, J. A., Remsberg, C. M., Takemoto, J. K., Davies, N. M., Rasco, B., \& Clary, C. (2010). Effect of dehydration on raspberries: polyphenol and anthocyanin retention, antioxidant capacity, and antiadipogenic activity. Journal of Food Science, 75(1), H5-H12. doi:10.1111/j.1750-3841. 2009.01383.x

Mitra, P., \& Meda, V. (2009). Optimization of microwave-vacuum drying parameters of saskatoon berries using response surface methodology. Drying Technology, 27(10), 1089-1096. doi:10.1080/07373930903221101

Mitra, P., Chang, K.-S., \& Yoo, D.-S. (2011). Kaempferol extraction from cuscuta reflexa using supercritical carbon dioxide and separation of kaempferol from the extracts. International Journal of Food Engineering, 7(4), 1-15. doi:10.2202/1556-3758.1768

Ozga, J. A., Saeed, A., \& Reinecke, D. M. (2006). Anthocyanins and nutrient components of saskatoon fruits (amelanchier alnifolia nutt.) Canadian Journal of Plant Science, 86(1), 193-197. doi:10.4141/P05-139. eprint: http: / / pubs . aic . ca/doi / pdf / 10 . 4141/P05-139

Patras, A., Brunton, N. P., O'Donnell, C., \& Tiwari, B. K. (2010). Effect of thermal processing on anthocyanin stability in foods; mechanisms and kinetics of degradation. Trends in Food Science \& Technology, 21 (1), 3-11. doi:10.1016/j.tifs.2009.07.004

Ratti, C. (2001). Hot air and freeze-drying of highvalue foods: a review. Journal of Food Engineering, 49(4), 311-319. doi:10.1016/S02608774(00)00228-4

Re, R, Pellegrini, N, Proteggente, A, Pannala, A, Yang, M, \& Rice-Evans, C. (1999). Antioxidant activity applying an improved abts radical cation decolorization assay. Free Radical Biology and Medicine, 26(9-10), 1231-1237. doi:10 . 1016 / S0891 - 5849(98) 00315-3

Sagar, V. R., \& Kumar, S. P. (2010). Recent advances in drying and dehydration of fruits and vegetables: a review. Journal of Food Science and Technology-Mysore, 47(1), 1526.

Subhasree, B., Baskar, R., Keerthana, R. L., Susan, R. L., \& Rajasekaran, P. (2009). Evaluation of antioxidant potential in selected green leafy vegetables. Food Chemistry, 115(4), 1213-1220. doi:10.1016/j . foodchem.2009.01.029

Wrolstad, R. E. (1993). Color and pigment analyses in fruit products. (Bulletin 624) Corvallis, Agricultural Experiment Station: Oregon State University. 